\title{
The effect of methadone and Opioid on BMI IN postmenopausal women
}

\section{Introduction}

Menopause is one of the normal stages of women's life. At this time, ovaries secrete less female hormones, especially estrogen., ${ }^{1,2}$ Men during menopause experience different symptoms that can affect the physiological, psychological and social aspects of a person's life. ${ }^{3}$ Among these problems can be hot flashes and increased blood pressure. $^{4}$

The symptoms of these symptoms are that the quality of life of women during menopause is severely threatened with increased symptoms. These symptoms are more severe in women who are overweight than Opioid and alcohol. As high alcohol intake and Opioid substances exacerbate menopausal symptoms, including flushing.

Deformation and body weight gain are the best symptoms of menopause in more than $90 \%$ of women, indicating severe changes in hormone levels. The current research is limited to healthy people, and since Opioid use and medications have a direct impact on body weight and menopausal symptoms, this study compared BMI and its effect on quality of life among three groups of healthy postmenopausal women, postmenopausal women with history of methadone use, and women who use oral contraceptives.

Materials and Methods: This cross-sectional study was performed on 90 age-old menopausal women aged 58 years. After obtaining permission from the Ethics Committee for research and written consent of the subjects, the subjects were divided into 3 groups: 30 healthy postmenopausal women and 30 postmenopausal women under methadone maintenance treatment as sampling Cluster samples were selected. In women with a history of drug use, snowball sampling was used. Entry condition, natural menopause without hysterectomy, passes more than one year from the last menstrual period and exit criteria. Having a history of any cardiovascular disease, diabetes, hypertension, Neurological and Cancer Disease, Hormonal Drug Use.

Questionnaire on the quality of life of menopause by Hildic and colleagues in Toronto, which has 32 questions and 4 dimensions of obsessive-compulsive, psychosocial, physical and sexual measurements. Measurement of height by age and body weight measurements were performed with SQA digital scales. Analysis and analysis Data were analyzed using SPSS16 software (statistical tests and one way ANOVA).

\section{Findings and discussion}

\section{In this present study, we examine relationship BMI and methadone}

The mean age of patients was 58 years old. Comparison of three groups regarding quality of life related to menopause did not show any significant difference among three groups $(p=704)$. However, the best the highest quality of life (Menopause Specific Quality of Life related to menopause) was observed in among healthy
Volume 9 Issue $6-2018$

\author{
Masumeh Ghazanfarpour \\ Evidence-Based Care Research Center, Mashhad University of \\ Medical Sciences, Iran
}

Correspondence: Masumeh Ghazanfarpour, Evidence-Based Care Research Center, Mashhad University of Medical Sciences, Mashhad I3944-9 I388, Iran, Tel +98-9I-3633-0656, Fax +98-5 I1859-73 I3, Email masumeh.ghazanfarpour@yahoo.com

Received: February 10, 2018| Published: December 27, 2018

women(38.75 \pm 8.30$)$, followed by Opioid addict (41.46 \pm 20.68$)$ and

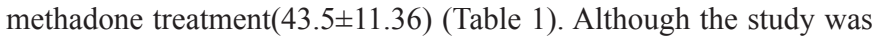
quasi-experimental, the findings are consistent with a multisite cohort study that showed the beneficial effect of methadone treatment and buprenorphine treatment on quality of life. ${ }^{3}$ Our study did not reach significant levels; this may be related small sample size.

There were a statistically significant difference among three groups regarding BMI $(\mathrm{p}<0.001)$. Health women had a higher BMI $(28.75 \pm 4.9)$ compared to Opioid addict $(24.31 \pm 4.28)$ and methadone treatment (22.03 \pm 2.7$)$. BMI was significantly lower in-patient under treatment with methadone compared to general population. Difference between group may related to behavioural eating stereotypes

This study is somewhat in consisting with Montazerifar et al. ${ }^{4}$ in which Methadone maintenance therapy increased significantly body weight from $61.4 \pm 14.4$ to $65.3 \pm 14.2 \mathrm{~kg}$. Also BMI increased significantly from $21.4 \pm 4.2$ to $23 \pm 5.6\left(\mathrm{~kg} / \mathrm{m}^{2}\right)$. Discrepancy between current findings with Montazerifar et al. ${ }^{4}$ study may be related to study design. The current study was a cross sectional while Montazerifar et al. ${ }^{4}$ used a before and after design with larger sample size. Future research should include before and after design or a before and after design as well as control group with larger sample size.

\section{Acknowledgments}

None.

\section{Conflicts of interest}

The author declares that they do not have any conflicts of interest.

\section{References}

1. Abdolahi F, Shabankhani B, Zargami M. Study of average age of menopause in Mazandaran province. Journal of Mazandaran University of Medical Sciences. 2005;14:61.

2. Williams RE, Levine KB, Kalilani L, Lewis J, Clark RV. Menopause-specific questionnaire assessment in US population-based study shows negative impact on health-related quality of life. Maturitas. 2009;62(2):153159 . 
3. Maremmani I, Pani PP, Pacini M, et al. Substance use and quality of life over 12 months among buprenorphine maintenance-treated and methadone maintenance-treated heroin-addicted patients. Journal of Substance Abuse Treatment. 2007;33(1):91-98.
4. Montazerifar F, Karajibani M, Lashkaripour K. Effect of methadone maintenance therapy on anthropometric indices in opioid dependent patients. International Journal of High Risk Behaviors \& Addiction. 2012;1(3):100 